\title{
ZEB1 Directly Inhibits GPX4 Transcription Contributing to ROS Accumulation in Breast Cancer Cells
}

\author{
yue wang ( $\nabla$ wangyue@nankai.edu.cn ) \\ Nankai University https://orcid.org/0000-0002-1570-5594 \\ xiao han \\ Nankai University College of Pharmacy \\ xianxian duan \\ Nankai University School of Medicine \\ zhanzhao liu \\ Nankai University School of Medicine \\ yaping long \\ Nankai University School of Medicine \\ chang liu \\ Nankai University School of Medicine \\ jing zhou \\ Nankai University School of Medicine \\ ning li \\ Tianjin University \\ junfang qin \\ Nankai University School of Medicine
}

\section{Research Article}

Keywords: Zinc finger E-box binding homeobox 1 (ZEB1), Reactive oxygen species (ROS), Glutathione peroxidase 4(GPX4)

Posted Date: April 26th, 2021

DOI: https://doi.org/10.21203/rs.3.rs-416905/v1

License: @ (i) This work is licensed under a Creative Commons Attribution 4.0 International License. Read Full License 


\section{Abstract}

Prior studies have noted that Zinc finger E-box binding homeobox 1 (ZEB1) is a master transcription regulator, affecting the expression of nearly 2,000 genes in breast cancer cells, especially in epithelial-mesenchymal transition (EMT) process. In this study, we found that ZEB1 directly regulated Glutathione peroxidase 4 (GPX4) transcriptions, which was closely related to tumor reactive oxygen species (ROS) metabolism. We selected two human breast cancer cell linesMDAMB-231 and MCF7 for ROS test, PCR, immunofluorescence, western blot, ChIP, luciferase assay and enzyme assay. The conclusions continue to be supported in mouse models and bioinformatics analysis. We found that ZEB1 directly bound to GPX4 promoter E-box motif (CANNTG) to inhibit GPX4 transcription, which contributing to ROS accumulation and tumorigenesis in breast cancer.

\section{Introduction}

Zinc finger E-box binding homeobox 1 (ZEB1), as an important member of zinc finger homologous domain transcription factor family, could bind on E-box and exert either promotion or inhibition of molecular transcription, regulating cancer cell differentiation and multidrug resistance ${ }^{1,2,3}$. Most of previous studies have only concentrated on its role in EMT. Little is known about ZEB1 affect ROS and oxidative stress metabolism.

Increased ROS is a typical malignancy feature in tumor ${ }^{4}$.The main endogenous sources of ROS are enzymes of the mitochondrial respiratory chain, NADPH oxidase (NOX), xanthineoxidase and dysfunctional endothelial nitric oxide synthases (NOS) ${ }^{5}$. ROS can be detrimental when produced intracellularly in high amounts and then the cells generally respond to ROS by up-regulating antioxidants such as superoxide dismutase (SOD) and glutathione peroxidase (GPx), which could initiate innate immune responses to tumors ${ }^{6}$. Vitamins $E$ and $C$ have also been proved to be ROS scavengers ${ }^{7,8}$. Cellular abnormal ROS level may promote the growth of cancer cells or may lead to cell death ${ }^{9}$.

Glutathione peroxidase 4(GPX4), as a member in ROS elimination system,could reduce the level of hydroperoxides by catalyzing glutathione ${ }^{10,11}$. Promoter region of GPX4 contains four E-box (CANNTG) motifs, which is reported to be regulated by ZEB1 ${ }^{12}$.

Thus, the aim of this study was to explore the influence of ZEB1 in cancer metabolic and oxidative stress cancer metabolism and further pursue for its possible mechanism.

\section{Results}

\section{ZEB1 promotes ROS levels in breast cancer cells}

Firstly, we use lentiviral-based vector pLV-H1-EF1a-puro to express shRNA (shZEB1) in MDA-MB-231 cells (Fig.S1).To access the impact of ZEB1 on cancer metabolism, we employed RNA sequencing (RNA-Seq) and GO enrichment analysis to find out deferentially expressed genes (DEGs) and enriched signaling pathways between shZEB1 and the wild-type in MDA-MB-231 cells. We identified 224 genes related to metabolic processes in 577 DEGs regulated by ZEB1 in biological process (BP)(Fig. 1A). Top 20 of BP DEGs were shown in Fig. 1B, and some of them(the circled) were significantly enriched to second messenger regulatory signaling, cell junction assembly, ERK-related pathways and platelet degranulation(Fig. 1B,P < 0.05), which were previously reported to be linked with oxidative stress injury ${ }^{15-18}$.The distribution dots and proportion of up-regulated and down-regulated genes of ZEB1 were shown in Fig. 1C and Fig. 1D, prominent FOXO6, CYGB, RAC2, SOD3 and BTG2 were explicitly related to ROS metabolism ${ }^{19,20-26}$ (Fig. 1C and Fig S3).

After ZEB1 knocked down by shZEB1 (231 sh) and 3 targeted sequencing siZEB1 (mcf7 \#1, mcf7 \#2and mcf7 \#3)(Fig S2), due to DCFH-DA fluorescence intensity decreasing, we found significant total ROS level reduction comparing to 
control (231 nc, mcf7 nc and mcf7 siNC for short as mcf7 \#nc) in MDA-MB-231 and MCF7 by flow cytometry. And the ROS reduction level was significant after ZEB1 knockdown, which was basically close to the effect of ROS scavengers, such as SOD(1000 U/mL), NAC(5 mM),TEMPO(1 mM) and Vitamin E(Vit E, $10 \mu \mathrm{g} / \mathrm{mL})$ (Fig. 2A and Fig S4, P<0.05). These achievements have raised the question: is the total ROS reduction due to less generation or increased scavenging pathways?

As mitochondrial ROS (mtROS) was the main sources of ROS(approximately $90 \%$ ) 27 , the ROS production of mitochondrial electron transport chain was simply and systematically detected ${ }^{5}$. However, mitochondrial respiratory chain complex (MRCC) I/III activity, which is main pathway of mtROS generation ${ }^{5}$, remained unchanged with ZEB1 being knocked down (Fig. 2B, $\mathrm{P}>0.05$ ). We also inspected NADPH system which is roughly responsible for the remaining ROS sources (approximately 10\%).We checked the expression of NOX1, NOX3 and NOX4 by qPCR, Vit E(10 $\mu \mathrm{g} / \mathrm{mL}) \mathrm{was}$ negative control, $\mathrm{H}_{2} \mathrm{O}_{2}(80 \mathrm{mmol} / \mathrm{L})$ was positive control and found that only NOX4 had a statistically significant change(Fig. $2 \mathrm{C}, \mathrm{P}<0.05$ ). Nevertheless, it could not be explained that NOX4 contributed much to ROS production. Therefore, it is recommended that the scavenging system for ROS should be to focus on in this study.

\section{ZEB1 directly restrains GPX4 promoter transcriptional activity by directly binding to E-box motif}

GPX4 was reported as a key player in ZEB1-related tumor progression ${ }^{28}$. To determine whether ZEB1 regulated GPX4 expression, we demonstrated GPX4 expression increased in both shZEB1 (231 sh) and siZEB1 subgroups (representative mcf7 \#2) on mRNA levels (Fig. 3A, P<0.05). The mRNA expressions of ZEB1 and GPX4 in breast cancer cells had a significant negative correlation, so is GPX4 regulated by ZEB1 transcriptional repression as a downstream target gene?

To answer that, we further analyzed GPX4 promoter sequence and found 4 E-box regions, which could be the binding sites of ZEB1(Fig. 3B). Then we performed ChIP assay and found both pairs of primers targeting GPX4 promoter E-box region could be immunoprecipitated by anti-ZEB1 with RT-PCR amplification analysis, suggesting the region containing the ZEB1 recognition sequence was in the GPX4 DNA. Columns represented that anti-ZEB1 levels bound to the GPX4 promoter was higher compared with anti-IgG in $231 \mathrm{nc}(\mathrm{Fig} .3 \mathrm{C}, \mathrm{P}<0.05)$. Luciferase assay further validated that ZEB1 directly inhibited the GPX4 transcription. We constructed pGL3 plasmids loading GPX4 promoter regions (E-box, CACCTG) or its mutated(MUT) fragment regions (GPX4-MUT, AAAAAA)(Fig. 3D).GV146-NC and GV146-ZEB1 were co-transfected in $231 \mathrm{nc}$ for $48 \mathrm{~h}$, then co-transfected by GPX4 promoter NC region plasmid with luciferase(luc) or GPX4 promoter MUT region plasmid with luc to test. We monitored significant decreases in luciferase activity only in over-expressed ZEB1 plasmid and transfected GPX4 promoter NC region plasmid with luc after 15 min and 45 min sessions(Fig. 3E, P<0.05). However, despite ZEB1 was over-expressed, GPX4 luciferase was stable in GPX4-MUT promoter transfected cells, which proved ZEB1 inhibited GPX4 promoter activity via binding E-box motifs (Fig. 3E).

\section{ZEB1 restrains GPX4 expression contributing to ROS accumulation in vitro}

Next, we examined the effect of ZEB1 on the down-regulation of GPX4 on related to basic functions such as ROS-driven oxidation and cell viability. First, the investigation in MDA-MB-231 cells treated by ShZEB1 (Fig. 4A) and MCF7 cells treated by siZEB1 (Fig. 4B) showed that both SOD and GPx activities increased after ZEB1 knockdown $(P<0.05)$. We inferred that ZEB1 increased ROS accumulation via prohibiting ROS eliminate pathway, that is enhanced SOD and GPX activities. In particular, the activity of GPx increased significantly after ZEB1 knockdown $(P<0.01)$, which strongly supported the finding that ZEB transitionally repressed GPX4.

Already it is known that, lipid alkoxyl(RO•) radicals (lipid ROS) is thought to contribute to ROS-dependent cell damage and death. We further strengthened ZEB1 restrains GPX4 expression through testing lipid ROS level. Lipid oxidation is one of the hallmarks of GPX4 expression level. C11-BODIPY as a lipid oxidation detection probe was used to detect the change of fluorescence intensity after MCF7 cells treated by siZEB1. Cells (mcf7 \#nc, mcf7 \#1, mcf7 \#2 and mcf7 \#3) were 
incubate with C11-BODIPY, then to be check on the flow cytometry. As shown in Fig. 4C, the mcf7 \#nc group showed highest fluorescence intensity compared with the siZEB1 groups (Fig. 4C, $P<0.05$ ). And the scavenging effect of lipid ROS of the siZEB1 groups might also curb the cytotoxicity(Fig. 4D, $P<0.05$ ), these results suggest siZEB1 got a strong cancer cell killing ability just like the up-regulating GPX4 removed ROS.

In addition, the researchers conclude that L-glutathione is the most important hydrophilic antioxidant that protects cells against exogenous and endogenous toxins, including ROS and reactive nitrogen species. In the GPx system, GPX4 catalyzes the $\mathrm{H}_{2} \mathrm{O}_{2}$ reduction, leading to GSH oxidation into GSSG (Fig. 4F). Therefore, detection of changes of the concentration of L-glutathione and GSSG after siZEB1 or shZEB1 in MCF7 or MDA-MB-231 cells is also necessary to indirectly determine the expression level of GPX4. We observed that loss of ZEB1 caused L-glutathione decreased and GSSG increased in MCF7 (Fig. 4E, P<0.05) and MDA-MB-231 cells (Fig. 4G, P < 0.05), and shZEB1 could decreased Lglutathione and increased GSSG with similar efficiency as ROS scavenger, such as SOD(1000 U/mL), NAC(5 mM), TEMPO $(1 \mathrm{mM})$ and Vit $E(10 \mu \mathrm{g} / \mathrm{mL})($ Fig. 4G, $P<0.05)$, the cells were treated in the same conditions as in Fig. 2A,suggesting that ZEB1 directly inhibited GPX4 expression contributing to ROS accumulation.

\section{ZEB1 restrains GPX4 expression and Vit E treatment facilitates GPX4 function in vivo}

Finally, we established cell line derived xenograft with MDA-MB-231 cells to determine whether ZEB1 silencing influenced tumor ROS and oxidative stress metabolism via GPX4 (Fig. 5A). Vit E eliminate ROS and suppress tumor growth ${ }^{30}$. We observed the tumor was smaller and lighter in shZEB1 group, and the trend has been sharper in groups which were maintained on diets with supplemented an antioxidant vitamin $\mathrm{E}$ (Fig. 5A-C, $\mathrm{P}<0.05$ ). Consistent with in vitro results, both SOD and GPX activity were increased in shZEB1 group mice, especially in Vit E feeding group(Fig. 5F, P< 0.05$)$. Increased expression of GPX4 was proved in mRNA and protein level after ZEB1 silenced, tendency could be augmented in Vit E feeding group(Fig. 5D, $\mathrm{P}<0.05$ and Fig. 5E).

Further, frozen sections of fresh tumor tissues were stained with fluorescent probe 013 ROS, and it was found that the down-regulated expression of ZEB1 decreased ROS intensity, and Vit E diet further reduced ROS in the tumor tissues (Fig. 6A, P < 0.05), which was consistent with the GPX4 protein level in Fig. 5D. Animal experiments further demonstrated that dispelling transcriptional inhibition of GPX4 by ZEB1 could effectively scavenge ROS, which might be one of the key mechanisms of inhibiting tumor growth.

In fact, we proved that ZEB1 could inhibit GPX4 transcription by binding to the E-box motifs and promote breast cancer progression by accumulating intracellular ROS. From the perspective of ROS clearance, Vit E could enhance GPX4 function to consume L-glutathione and eliminate excess intracellular ROS (Fig. 6B), which might contribute to the progression of breast cancer. In the end, we analyzed the role of ZEB1 and GPX4 in the survival of breast cancer patients by PROGgenev $2^{29}$. Correspondingly with the above conclusions, a higher ZEB1 mRNA expression was associated with shorten relapse-free survival in breast cancer patients, and a higher GPX4 mRNA expression did the opposite (Fig. 6C, P< 0.05 or $\mathrm{P}<0.01)$. Or, the major achievements of this study included that we strengthened ZEB1 is a promising metabolism therapeutic target.

\section{Discussion}

The present study provided insight into that ZEB1 repressed the scavenging ROS generation by directly repressing GPX4 transcription and promoted tumor progression high expression of ZEB1 and low expression off GPX4 were the adaptive mechanisms used by the malignancy breast cancer to withstand environmental pressures. Meanwhile, Vit E enhanced the expression and function of GPX4 in breast cancer. 
A re-evaluation of ZEB1 have been warranted that its pleiotropic functions in physiological and pathological conditions, not solely limited to cell invasion and dissemination ${ }^{31}$. The function and the mechanisms of ZEB1 are more complex than it was appreciated once ${ }^{32-34}$. ROS implicated in ionizing radiation-induced EMT via activation of transcription factors, including ZEB1 ${ }^{35}$, which is a favorable target of $\mathrm{H}_{2} \mathrm{O}_{2}{ }^{36}$. Modulation of oxidative stress has been reported as an anticancer strategy 37,38 . In particular about lipoperoxide, research on the lipid GPX4 has become more widespread ${ }^{39}$. We aimed to investigate how ZEB1 regulated GPX4 expression and how it affected the malignancy of breast cancer cells.

GPX4 is an essential regulatory inhibitor of ferroptotic cancer cell death ${ }^{40}$, and ferroptosis is a mode of cell death involving the production of iron-dependent ROS ${ }^{28}$. However, GPX4 knockout mice are embryologically lethal ${ }^{41}$. Some small molecule inhibitors could prevent cell death in response to GPX4 deletion, which could trigger acute renal failure in mice ${ }^{42}$. Small intestinal epithelial cells in Crohn's disease exhibit impaired GPX4 function and signs of lipid peroxidation ${ }^{43}$. These data confirmed that GPX4 was benefit to disease therapy. SOD and GPx activities were elevated after ZEB1 silenced, and ZEB1 could transcriptionally suppress the expression of GPX4. It is reasonably expected that Vit $E$, as ROS scavenger, showed no additional effects on ZEB1 silencing. Vit E abolished DHA docosahexaenoic acid (DHA) effects on GPx inhibition, and DHA enhanced the activity of several anticancer drugs through an oxidative mechanism ${ }^{30}$. GPx is an important peroxidase that exists widely in the body. Selenium (Se) is a component of GPx enzyme system, which can catalyze the transformation of glutathione (GSH) into GSSG, reduce toxic peroxide to non-toxic hydroxyl compounds, and promote the decomposition of $\mathrm{H}_{2} \mathrm{O}_{2}$, so as to protect the structure and function of cell membrane from the interference and damage of oxides. Findings obtained by these in vitro and in vivo studies strongly suggest that the selenoenzyme GPX4 is a critical factor for breast cancer cells survival which is highly sensitive to Vit E status. It suggests the specific molecular mechanism existing between Vit E and GPX4 circuit and remains to be further explored.

Given that cancer cells must be able to cope with high oxidative stress to promote metastasis, ROS exist as carcinogenic factor $^{4}$. Whereas when cellular ROS level reach the "threshold" that can lead to cell death, ROS exert a cytotoxic effect to limit cancer progression ${ }^{9}$. Our exploration was mostly conducted on the basis of ZEB1 silencing, intracellular ROS level remained at the high level of its long-term oxidative adaptation, but did not reach the threshold. At the early stage of the experiment, we detected the effect of ZEB1 on ROS production, and the activity of MRCC I/III was not interfered by siZEB1. In addition to MRCC, ROS production pathways also include NADPH oxidase and xanthine oxidase, etc ${ }^{5}$. ROS can be detrimental (it is then referred to as "oxidative and nitrosative stress") when produced in high amounts in the intracellular compartments and cells generally respond to ROS by up-regulating antioxidants such as SOD and catalase (CAT), GPx and GSH that protects them by converting dangerous free radicals to harmless molecules (i.e. water). Therefore, the reason why ZEB1 up-regulated ROS level in breast cancer is not only through GPX4 inhibition of the clearance pathway, but also whether there are generative pathways other than MRCC I/III or NOX1, NOX3 and NOX4 involved remains to be further studied.

In conclusion, ZEB1, as a universal transcription factor, could not only regulate EMT, but inhibit GPX4 transcription via binding to E-box motif. It is significant to consider that the ZEB1/GPX4 axis has a therapeutic effect on breast cancer metabolism.

\section{Materials And Methods}

\section{Cell culture}

All our experiments were conducted in two human breast cancer cell lines MDA-MB-231 and MCF7, which were purchased from ATCC. Cells were maintained in RPMI 1640; with $10 \%$ fetal bovine serum in $5 \% \mathrm{CO}_{2}$ humidified incubatorat $37^{\circ} \mathrm{C}$. 
Cells were transfected siRNA (RiboBio, china) using LipoJet ${ }^{\mathrm{TM}}$ Reagent (SignaGen, USA) according to the manufacturer's protocol. Vitamin E (DL-a-tocopheryl acetate, Vit E for short, Med Chem Express, USA).

\section{RNA-sequencing (RNA-Seq)}

The samples of MDA-MB-231 cells and the cells knocked down ZEB1 $(n=3)$ were sent to Beijing Genomics institution for RNA-Seq. The analysis was based on Bioconductor packages "cluster Profiler" soft ware.

\section{Plasmid construction and Generation of lentiviruses}

The human complementary DNA fragment encoding full-length ZEB1 was cloned into GV146 (Shanghai Gene chem, China), and the vector was transfected to MDA-MB-231 cells for $48 \mathrm{~h}$ to over-express ZEB1. The lentiviral-based vector pLV-H1-EF1a-puro (Biosettia, San Diego, USA) was used to express shRNA in MDA-MB-231 cells. Lentiviruses were generated by transfecting subconfluent HEK293T cells with lentiviral vectors and packaging plasmids by calcium phosphate transfection. Viral supernatants were collected after $48 \mathrm{~h}$ following transfection, centrifuged and filtered through 0.45-mm filters (Millipore, MA, USA).

\section{RT-PCRand qPCR}

Total RNA was isolated using TRIzol reagent (CWBIO, China). cDNA was obtained using EasyScript First-Strand cDNA Synthesis SuperMix (TransGen, China). $50 \mathrm{ng}$ cDNA was used to perform qPCR with UltraSYBR Mixture (CWBIO, China). Gene expression was normalized to control using $2^{-\Delta \Delta C t}$ method. Relative expression values less than 0.001 were considered extremely low/undetectable. Primer sequences are listed in Tables 1.

\section{Western blot(WB)}

Total protein $\left(20 \mathrm{mg}\right.$ ) from each sample (including cells and tissues) was mixed with loading buffer and heated at $95^{\circ} \mathrm{C}$. Proteins were separated in $12 \%$ SDS-PAGE, transferred to polyvinylidene difluoride membranes (Millipore, USA) and probed with primary antibodies to ZEB1 (1:1000, Abcam), GPX4 (1:1000, Bioss) and actin (1:500, Santa Cruz Biotechnology) at $4{ }^{\circ} \mathrm{C}$ overnight. Following secondary horseradish peroxidase-conjugated antibody incubation (1:5000, Tianjin Sungene Biotech), membranes were exposed with enhanced chemiluminescence agent (Millipore, USA) using TANON image software (Beijing YuanPingHao Biotech).

\section{Total ROS and lipid ROS measurement}

Intracellular total ROS level was examined using fluorescent dyes 2,7-Dichlorodi-hydrofluoresce in diacetate (DCFH-DA) ${ }^{14}$ as probes. The cells were trypsinized and incubated with DCFH-DA-containing serum-free medium, the intensity of the 2,7Dichlorodi-hydrofluoresce was detected by flow cytometry. Lipid ROS level was evaluated by cells incubating with the lipid peroxidation sensor C11-BODIPY (5 mM) for $30 \mathrm{~min}$, the fluorescent intensity was also for flow cytometry study.

\section{Measurements of MRCC I/III, SOD, GPx, L-Glutathione and GSSG}

Activities of Mitochondrial respiratory chain complex (MRCC I/III), total SOD (T-SOD, SOD for short) and glutathione peroxidase (GSH-Px, GPx for short) were employed for biochemical detection of cell lysates or the supernatant of tumor tissue homogenate (Beyotime, Shanghai, China). Concentrations of total L-Glutathione and GSSG were measured in cell lysates (Beyotime, Shanghai, China).

\section{Cell viability assay}

Cells were seeded into 96 -well plate $\left(1 * 10^{4}\right.$ cells per well) and incubated for overnight at $37^{\circ} \mathrm{C}$. The cells were incubated 
with ROS scavengers at different concentrations for $24 \mathrm{~h}$ at $37^{\circ} \mathrm{C}$, and then analyzed via the standard CCK-8 assay (ab228554, abcam).

\section{Chromatin immunoprecipitation (ChIP) assay}

EZ-Magna ChIP ${ }^{\text {TM }}$ A/G Chromatin Immunoprecipitation Kit (MERCK, Germany) was used. Antibodies used for immunoprecipitation were goat anti-rabbit ZEB1, isotype IgG and RNA polymerase II antibody (MERCK, Germany) as negative control and positive control, respectively. Primers for ChIP assay are listed in Tables 2. End-point PCR was conducted to detect targeted DNA.

\section{Luciferase assay}

GPX4 promoter or mutant binding sites promoter sequences were cloned into pGL3 luciferase promoter vectors (General Biosystems) using the restriction sites of the enzymes Kpnl and Xhol. MDA-MB-231 cells were co-transfected with the wild-type or mutant human GPX4 promoters and ZEB1 expression plasmid or control in 6-well plates. Cells were prepared after $48 \mathrm{~h}$ following transfection, and incubated with D-Luciferin, luciferase activity was measured according to the manufacturer's protocols.

\section{Xenograft tumor growth in nude mice}

As approved by the ethics committee for animal use in Nankai University. The mice were randomized into 3 groups ( $n=3$ ), and MDA-MB-231 cells $\left(2 * 10^{6}\right)$ transfected with shCtrl(231 nc) or shZEB1(231 sh) were implanted subcutaneously(SQ) into the mammary fat pads of female BALB/c nude mice respectively. In the second day of implanting tumor cells, 0.5 $\mathrm{g} / \mathrm{kg}$ Vitamin E was supplemented in the food and fed to the only one group until mice sacrificing, and the others were fed without vitamin $\mathrm{E} \operatorname{diet}^{13}$. Tumors were monitored every 3 days by caliper, and tumor volume was calculated as $0.5 \times$ length $\times$ width $\times$ width. Then the 9 tumors were removed and the fresh tissues were extracted for nucleic acid, protein, homogenate and frozen sections.

\section{Frozen section immunofluorescence}

Frozen sections were made from fresh mice implant tumors (within 1 hour after surgical resection) (Thermo Cryotome E), spun dry and circled around the tissues with a marking pen. Added containing fluorescent probe 013 ROS of dye solution (BBoxiProbe ${ }^{\circledR}$ tissues active oxygen detection kit, BB-470512, Bestbio, Shanghai, China) within the circle, and incubated and shook for $30 \mathrm{~min}$ at $37^{\circ}$ out of light. After the sections were dried, DAPI dye was added into the circle and incubated at room temperature in the dark for $10 \mathrm{~min}$. Antifade Mounting Medium (Beyotime Shanghai, China) was used to seal the sections, which were observed and imaged under a fluorescence confocal microscope (FV1000, Olympus) (013 ROS probe is a red fluorescent ROS probe with a maximum excitation/emission wave length of 510/610 nm).

\section{Statistical Analysis}

Data analyses was conducted in GraphPad5. All data from at least 3 experiments was averaged by \pm SEM.The difference between two groups was analyzed using the Student's $t$-test. The difference among three or more groups was evaluated by one-way ANOVA, that followed by the Dunnett' test.

\section{Declarations}

\section{Acknowledgments}

We thank Professor Phillip Bryant (Nankai University) for linguistic support. This work was funded by grants from the Natural Science Foundation of China (No.31770968 and 31800661), the Open Project Program of Key Laboratory for 
Tumor Precision Medicine of Shaanxi Province (KLTPM-SX2017-A3\& KLTPM-SX2018-B1) and the Open Project Program of the Chongqing TCM key Laboratory for Metabolic Disease (Chongqing Medical University).

\section{Disclosure Statement}

The authors declare no conflicts of interest for this article.

\section{References}

1. Krebs AM et al (2017) The EMT-activator Zeb1 is a key factor for cell plasticity and promotes metastasis in pancreatic cancer. Nat Cell Biol 19:518-529. doi:10.1038/ncb3513

2. Brabletz S, Brabletz T (2010) The ZEB/miR-200 feedback loop-a motor of cellular plasticity in development and cancer? EMBO Rep 11:670-677. doi:10.1038/embor.2010.117

3. Jiang Y, Wang D, Ren H, Shi Y, Gao Y (2018) Oncogenic HBXIP enhances ZEB1 through Sp1 to accelerate breast cancer growth. Thorac Cancer 9:1664-1670. doi:10.1111/1759-7714.12878

4. Feng $S$ et al (2019) Multi-dimensional roles of ketone bodies in cancer biology: Opportunities for cancer therapy. Pharmacol Res 150:104500. doi:10.1016/j.phrs.2019.104500

5. Poprac P et al (2017) Targeting Free Radicals in Oxidative Stress-Related Human Diseases. Trends Pharmacol Sci 38:592-607. doi:10.1016/j.tips.2017.04.005

6. Jia M et al (2020) Redox homeostasis maintained by GPX4 facilitates STING activation. Nat Immunol 21:727-735. doi:10.1038/s41590-020-0699-0

7. Wu D, Yotnda P (2011) Production and detection of reactive oxygen species (ROS) in cancers. J Vis Exp. doi:10.3791/3357

8. Lee GY, Han SN The Role of Vitamin E in Immunity. Nutrients10, doi:10.3390/nu10111614 (2018)

9. Trachootham D, Alexandre J, Huang P (2009) Targeting cancer cells by ROS-mediated mechanisms: a radical therapeutic approach? Nat Rev Drug Discov 8:579-591. doi:10.1038/nrd2803

10. Hayes JD, Dinkova-Kostova AT, Tew KD (2020) Oxidative Stress in Cancer. Cancer Cell 38:167-197. doi:10.1016/j.ccell.2020.06.001

11. Ursini F, Maiorino M (2020) Lipid peroxidation and ferroptosis: The role of GSH and GPx4. Free Radic Biol Med 152:175-185. doi:10.1016/j.freeradbiomed.2020.02.027

12. Zhang $\mathrm{J}$ et al (2017) ZEB1 induces ER-alpha promoter hypermethylation and confers antiestrogen resistance in breast cancer. Cell Death Dis 8:e2732. doi:10.1038/cddis.2017.154

13. DIAO QX (2016) J. Z. Z., T. ZHAO. Vitamin E promotes breast cancer cell proliferation by reducing ROS production and p53 expression. Eur Rev Med Pharmacol Sci 20:2710-2717

14. Huang $C$ et al. Interaction Mechanisms Between the NOX4/ROS and RhoA/ROCK1 Signaling Pathways as New Antifibrosis Targets of Ursolic Acid in Hepatic Stellate Cells. Front Pharmaco/10, 431, doi:10.3389/fphar.2019.00431 (2019)

15. Sies H, Berndt C, Jones DP (2017) Oxidative Stress. Annu Rev Biochem 86:715-748. doi:10.1146/annurev-biochem061516-045037

16. Jiang $L$, Shestov AA, Swain P Reductive carboxylation supports redox homeostasis during anchorage-independent growth. Nature532, 7598, doi:10.1038/nature17393 (2016)

17. Schafer ZT, Grassian AR, Song L Antioxidant and Oncogene Rescue of Metabolic Defects Caused by Loss of Matrix Attachment. Nature3; 461(7260), 109-113, doi:10.1038/nature08268 (2009) 
18. Zhang XF et al (2020) Andrographolide attenuates oxidative stress injury in cigarette smoke extract exposed macrophages through inhibiting SIRT1/ERK signaling. Int Immunopharmacol 81:106230.

doi:10.1016/j.intimp.2020.106230

19. Aiping J.etal. Downregulation of FOXO6 alle via teshypoxia-induced apoptosis and oxidative stress in cardiomyocytes by enhancing Nrf2 activation via upregulation of SIRT6. Journal of Bioenergetics and Biomembranes52, 409-419, doi: 10.1007/s10863-020-09856-2 (2020)

20. YichaoX (2020) et al. High-fat diet selectively decreases bone marrow $\operatorname{lin}^{-} / \mathrm{CD} 117^{+}$cell population in aging mice through increased ROS production. J Tissue Eng Regen Med 14:884-892. doi:10.1002/term.3047

21. ElisaB R et al. The Antioxidative Role of Cytoglobin in Podocytes: Implications for a Role in Chronic Kidney Disease. Antioxidants \& redox signaling32, 1155-1171, doi: 10.1089/ars.2019.7868 (2020)

22. JenniferL, Larson-Casey et al (2018) Macrophage Rac2ls Required to Reduce the Severity of Cigarette Smokeinduced Pneumonia. Am J Respir Crit Care Med 198:1288-1301. doi:10.1164/rccm.201712-23880C

23. Jung-A (2013) Choi\&InKyoung, Lim. TIS21/BTG2 inhibits invadopodia formation by downregulating reactive oxygen species level in MDA-MB-231cells. J Cancer Res Clin Oncol 139:1657-1665. doi:10.1007/s00432-013-1484-3

24. Weifeng $T$ et al (2021) TMT-based quantitative proteomics reveals suppression of SLC3A2 and ATP1A3 expression contributes to the inhibitory role of acupuncture on airway inflammation in an OVA-induced mouse asthma model. Biomed Pharmacother 134:111001. doi:10.1016/j.biopha.2020.111001

25. Dario C, Altieri (2019) Mitochondrial dynamics and metastasis. Cell Mol Life Sci 76:827-835. doi:10.1007/s00018018-2961-2

26. David S (2018) Park\&Glenn I, Fishman.SCN5A: the greatest HITS collection. J Clin Invest 128:913-915. doi:10.1172/JCI99927

27. Dan Dunn J, Alvarez LA, Zhang X, Soldati T (2015) Reactive oxygen species and mitochondria: A nexus of cellular homeostasis. Redox Biol 6:472-485. doi:10.1016/j.redox.2015.09.005

28. Viswanathan VS et al (2017) Dependency of a therapy-resistant state of cancer cells on a lipid peroxidase pathway. Nature 547:453-457. doi:10.1038/nature23007

29. < PROGgeneV2 enhancements on the existing database.pdf>.

30. Vibet S et al (2008) Sensitization by docosahexaenoic acid (DHA) of breast cancer cells to anthracyclines through loss of glutathione peroxidase (GPx1) response. Free Radic Biol Med 44:1483-1491.

doi:10.1016/j.freeradbiomed.2008.01.009

31. Goossens S, Vandamme N, Van Vlierberghe P, Berx G (2017) EMT transcription factors in cancer development reevaluated: Beyond EMT and MET. Biochim Biophys Acta Rev Cancer 1868:584-591.

doi:10.1016/j.bbcan.2017.06.006

32. Zhang P, Sun Y, Ma L (2015) ZEB1: at the crossroads of epithelial-mesenchymal transition, metastasis and therapy resistance. Cell Cycle 14:481-487. doi:10.1080/15384101.2015.1006048

33. Antonio AP, Jennifer JL, Kristen JT (2003) LK. Regulation of Smad signaling through a differential recruitment of coactivators and corepressors by ZEB proteins. EMBO J 22:2453-2462

34. Madany M, Thomas T, Edwards LA (2018) The Curious Case of ZEB1. Discoveries (Craiova 6:e86. doi:10.15190/d.2018.7

35. Lee SY et al. Induction of metastasis, cancer stem cell phenotype, and oncogenic metabolism in cancer cells by ionizing radiation. Mol Cancer16, 10, doi:10.1186/s12943-016-0577-4 (2017)

36. Luanpitpong $S$ et al (2018) Reactive oxygen species mediate cancer stem-like cells and determine bortezomib sensitivity via Mcl-1 and Zeb-1 in mantle cell lymphoma. Biochim Biophys Acta Mol Basis Dis1864:3739-3753. doi:10.1016/j.bbadis.2018.09.010 
37. Hecht F et al (2016) The role of oxidative stress on breast cancer development and therapy. Tumour Biol 37:42814291. doi:10.1007/s13277-016-4873-9

38. Gorrini C, Harris IS, Mak TW (2013) Modulation of oxidative stress as an anticancer strategy. Nat Rev Drug Discov 12:931-947. doi:10.1038/nrd4002

39. Forcina GC, Dixon SJ GPX4 at the Crossroads of Lipid Homeostasis and Ferroptosis. Proteomics, e1800311, doi:10.1002/pmic.201800311 (2019)

40. Yang WS et al. Regulation of ferroptotic cancer cell death by GPX4. Cel/156, 317-331, doi:10.1016/j.cell.2013.12.010 (2014)

41. Carlson BA et al (2016) Glutathione peroxidase 4 and vitamin E cooperatively prevent hepatocellular degeneration. Redox Biol 9:22-31. doi:10.1016/j.redox.2016.05.003

42. Friedmann Angeli JP et al (2014) Inactivation of the ferroptosis regulator Gpx4 triggers acute renal failure in mice. Nat Cell Biol 16:1180-1191. doi:10.1038/ncb3064

43. Mayr L et al (2020) Dietary lipids fuel GPX4-restricted enteritis resembling Crohn's disease. Nat Commun 11:1775. doi:10.1038/s41467-020-15646-6

\section{Tables}

\section{Table 1:}

PCR primers used in this study.

\begin{tabular}{|llllll|}
\hline mRNA & $\begin{array}{l}\text { mRNA } \\
\text { name }\end{array}$ & $\begin{array}{l}\text { Gene } \\
\text { ID }\end{array}$ & Forward primer & Reverse primer & $\begin{array}{c}\text { Amplicon } \\
\text { size (bp) }\end{array}$ \\
\hline ZEB1 & $\begin{array}{l}\text { Zinc finger } \\
\text { E-box } \\
\text { binding } \\
\text { homeobox } \\
1\end{array}$ & 6935 & CCTGTCCATATTGTGATAGAGGC & ACCCAGACTGCGTCACATGT & 147 \\
\hline NOX1 & $\begin{array}{l}\text { NADPH } \\
\text { oxidase 1 }\end{array}$ & 27035 & TTGTTTGGTTAGGGCTGAATGT & GCCAATGTTGACCCAAGGATTTT & 106 \\
\hline NOX3 & $\begin{array}{l}\text { NADPH } \\
\text { oxidase 3 }\end{array}$ & 50508 & CGTGGCGCATTTCTTCAACC & GCTCTCGTTAGGGGTGTTGC & 106 \\
\hline NOX4 & $\begin{array}{l}\text { NADPH } \\
\text { oxidase 4 }\end{array}$ & 50507 & TGTGCCGAACACTCTTGGC & ACATGCACGCCTGAGAAAATA & 136 \\
\hline GPX4 & $\begin{array}{l}\text { glutathione } \\
\text { peroxidase } \\
4\end{array}$ & 2879 & GAGGCAAGACCGAAGTAAACTAC & CCGAACTGGTTACACGGGAA & 100 \\
\hline $\begin{array}{l}\text { B- } \\
\text { actin }\end{array}$ & $\begin{array}{l}\text { beta } \\
\text { cytoskeletal } \\
\text { actin }\end{array}$ & 60 & CATGTACGTTGCTATCCAGGC & CTCCTTAATGTCACGCACGAT & 250 \\
\hline
\end{tabular}

\section{Table S2:}

PCR primers of GPX4 promoter regions used for ChIP assay. 


\begin{tabular}{|llll|}
\hline Gene & Forward primer & Reverse primer & Amplicons size (bp) \\
\hline GPX4 Primer 1 & GGGGTAGAGTGGCTCACA & TTAGTAGATACGGGCTTTCA & 119 \\
\hline GPX4 Primer 2 & GTGATGAGAAGCAAACCCAA & GGATTACACGAGCGAGCC & 228 \\
\hline
\end{tabular}

\section{Figures}

A

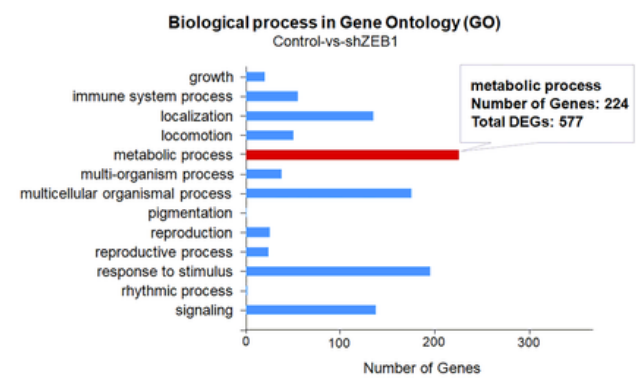

B

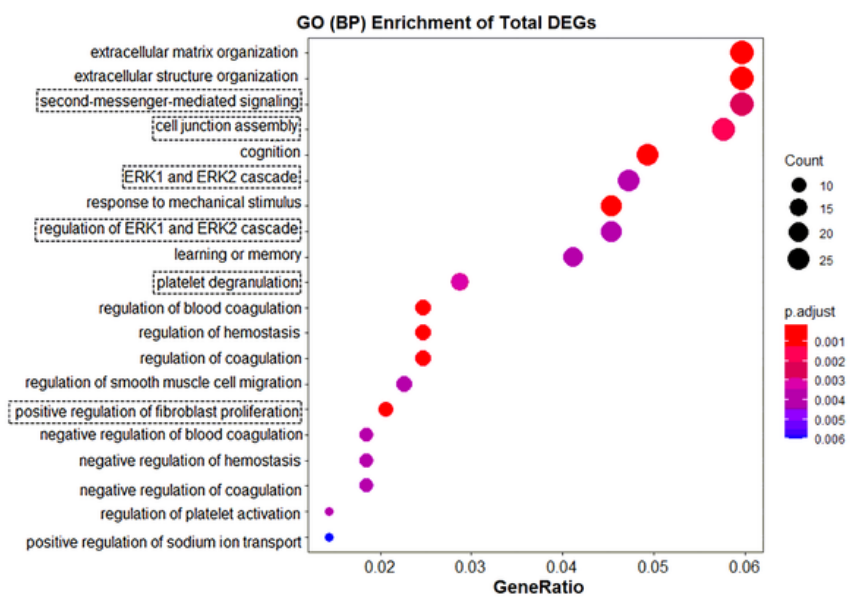

C

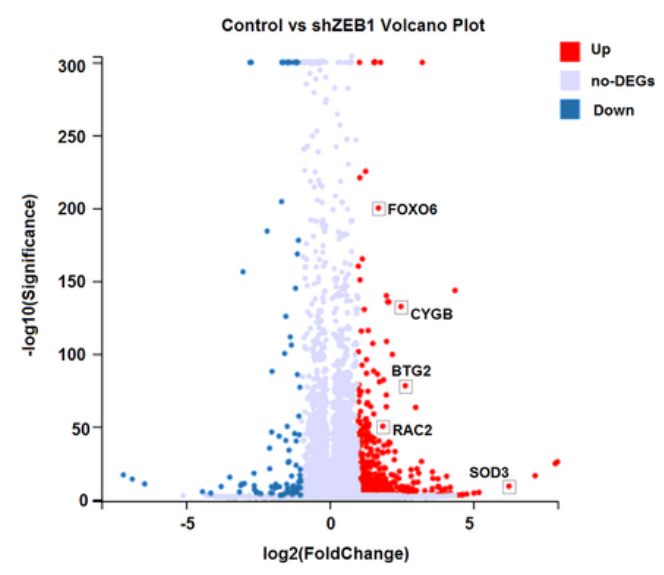

D

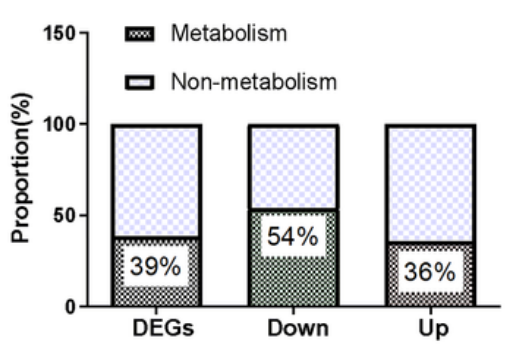

\section{Figure 1}

ZEB1 promotes ROS levels in RNA-Seq ( $n=3)$. A and B, metabolic process proportion and GO term enrichment of the top 20 selected DEGs in biological process (BP) was presented after shZEB1. C and D, proportion of up-regulated and downregulated genes in DEGs and some of them are involved in ROS regulation. 
A

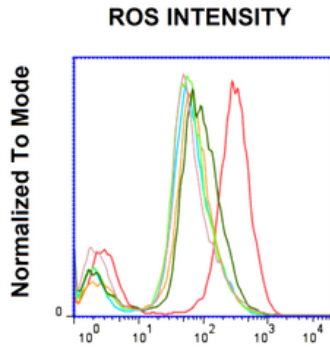

FL1-H:DCFH-DA

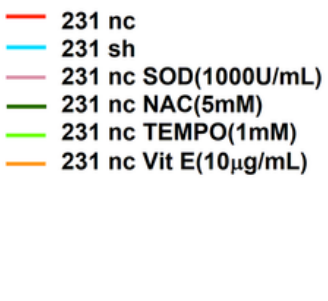

$231 \mathrm{nc}$ NAC (5mM)

$231 \mathrm{nc}$ TEMPO(1 $1 \mathrm{mM})$

$231 \mathrm{nc}$ Vit $\mathrm{E}(10 \mu \mathrm{g} / \mathrm{mL})$

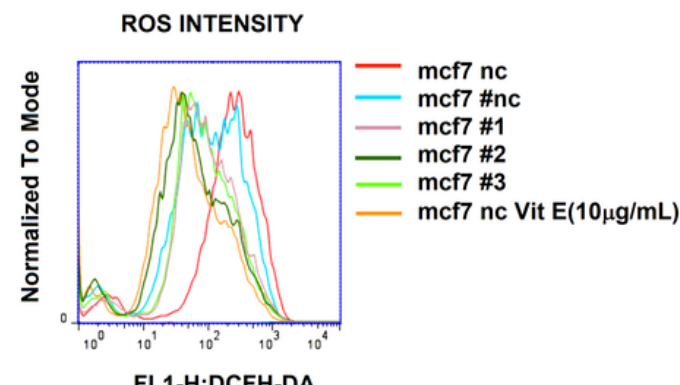

B
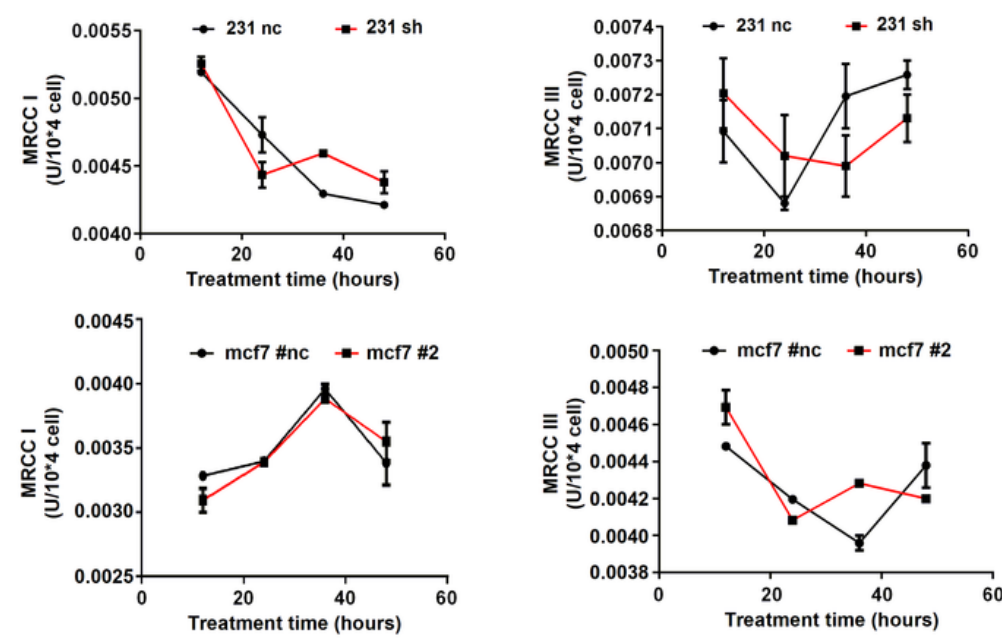

C
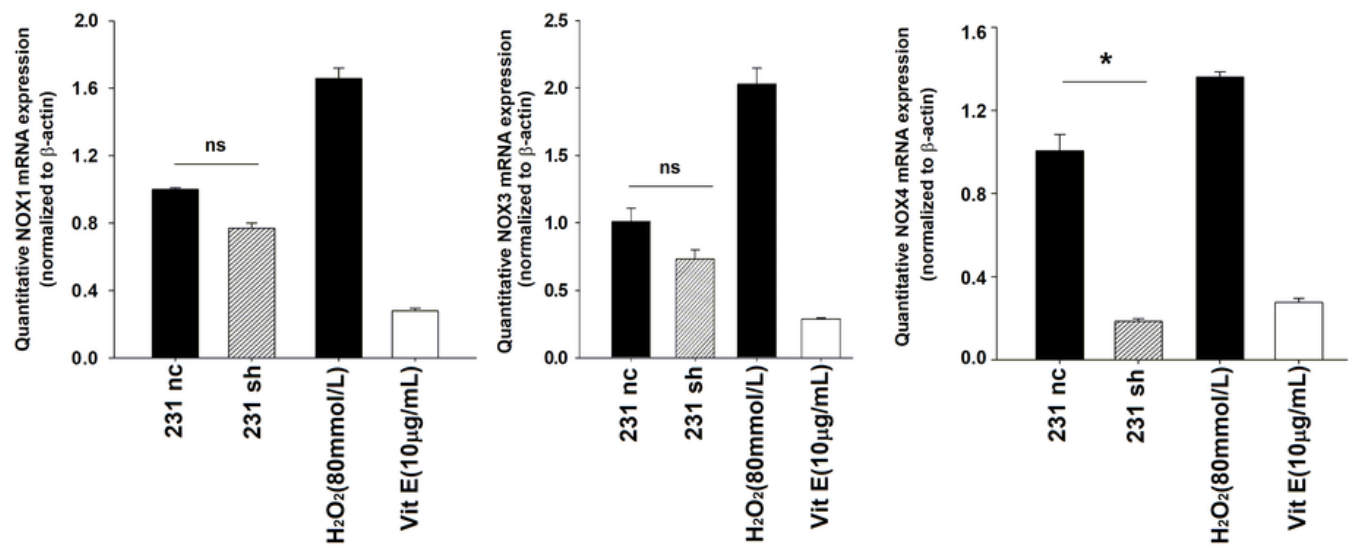

Figure 2

ZEB1 promotes ROS levels in vitro. A, ROS intensity was detected with DCFH-DA after shZEB1, siZEB1 as well as ROS scavengers in MDA-MB-231 and MCF7 cells. B, MRCC I/III activities were measured in MDA-MB-231 and MCF7 cells. Mean \pm S.E. of each group was presented $(n=6),{ }^{*}>0.05$. C $\triangle N O X 1 \otimes N O X 3 \otimes N O X 4$ mRNA expression was detected to measure ROS generation with ZEB1 silenced in MDA-MB-231 cells. $\mathrm{H} 2 \mathrm{O} 2$ and Vit $\mathrm{E}$ were used as a positive and negative control to induce ROS. Data are represented as means \pm S.E. in triplicate experiments, ${ }^{*} P<0.05$. NS: no significance. 
A
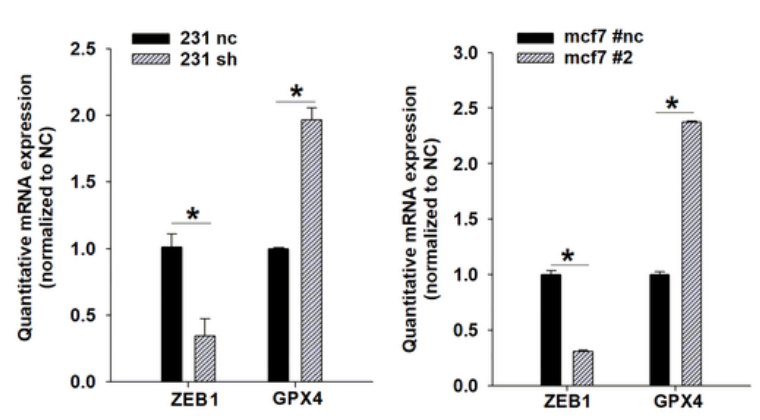

B

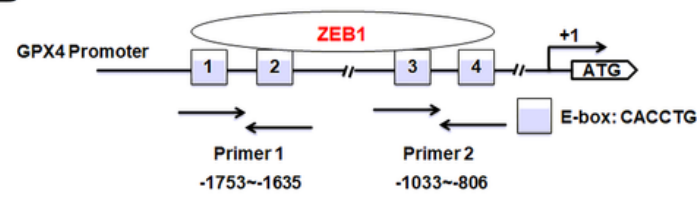

C

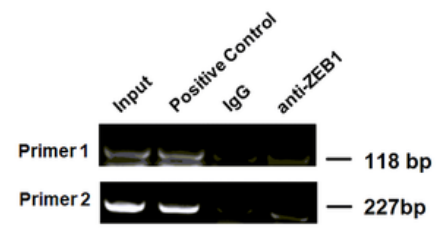

IP

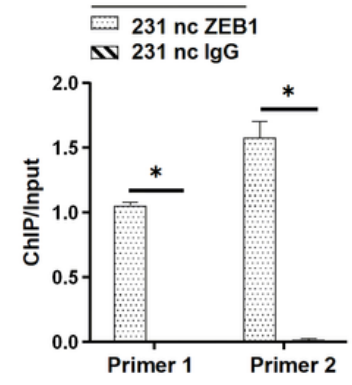

Positive Control: Anti-RNA polymerase II

IP: Anti-ZEB1

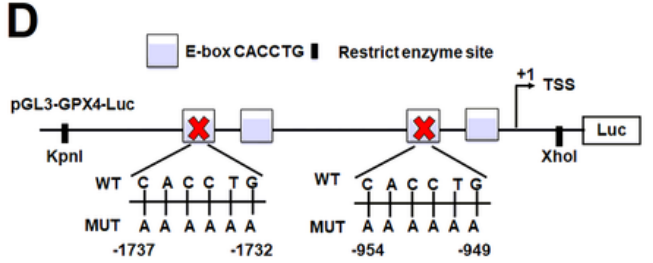

$\mathbf{E}$

Tranfection $48 \mathrm{~h} /+$ luc $15 \mathrm{~min}$

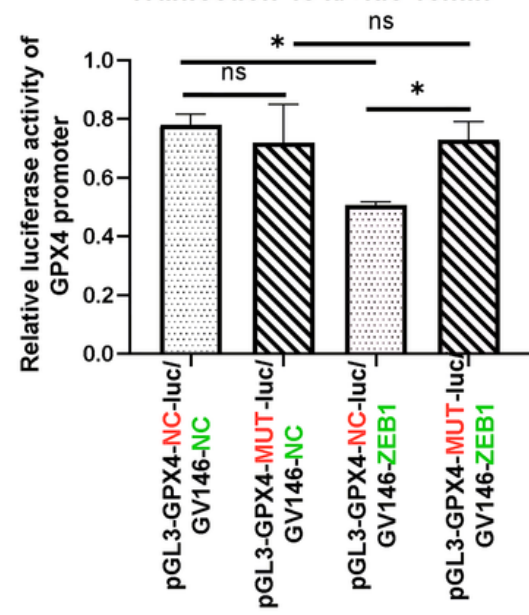

Tranfection $48 \mathrm{~h} /$ +luc $45 \mathrm{~min}$

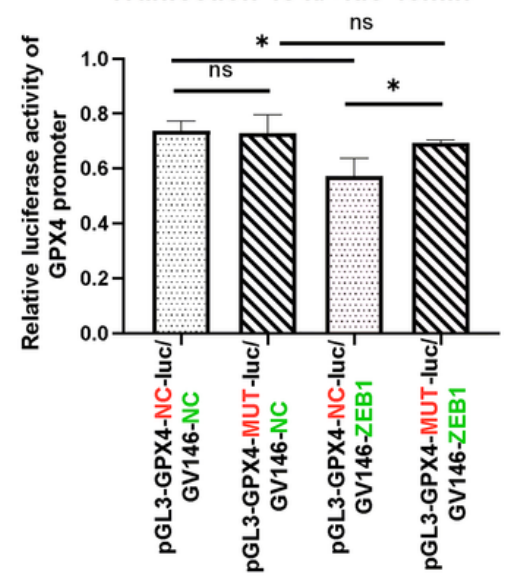

Figure 3

ZEB1 directly restrains GPX4 promoter transcriptional activity. A, GPX4 mRNA expression was increased in shZEB1 and siZEB1 cell lines. B, nucleotide sequence for human gene GPX4 promoter and E-box locations in GPX4 promoter region was exhibited. C, RT-PCR was used to detect gene abundance in different groups, which were immunoprecipitated by antiZEB1 antibody in MDA-MB-231 cells. D, GPX4 promoter region and its mutated fragment region (GPX4-MUT) were cloned into pGL3 plasmid. E, MDA-MB-231 cells were transfected with GPX4-NC promoter or GPX4-MUT then transfected with GV146-NC or GV146-ZEB1 over-expression vectors, and relative luciferase activity were measured, and normalized to promoter transfected alone group, respectively. Data are represented as means \pm S.E. in triplicate experiments, ${ }^{\star} P<0.05$. 
A

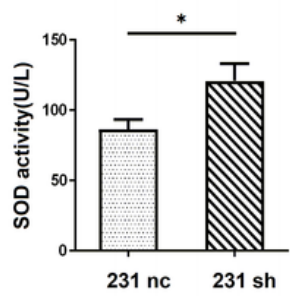

C
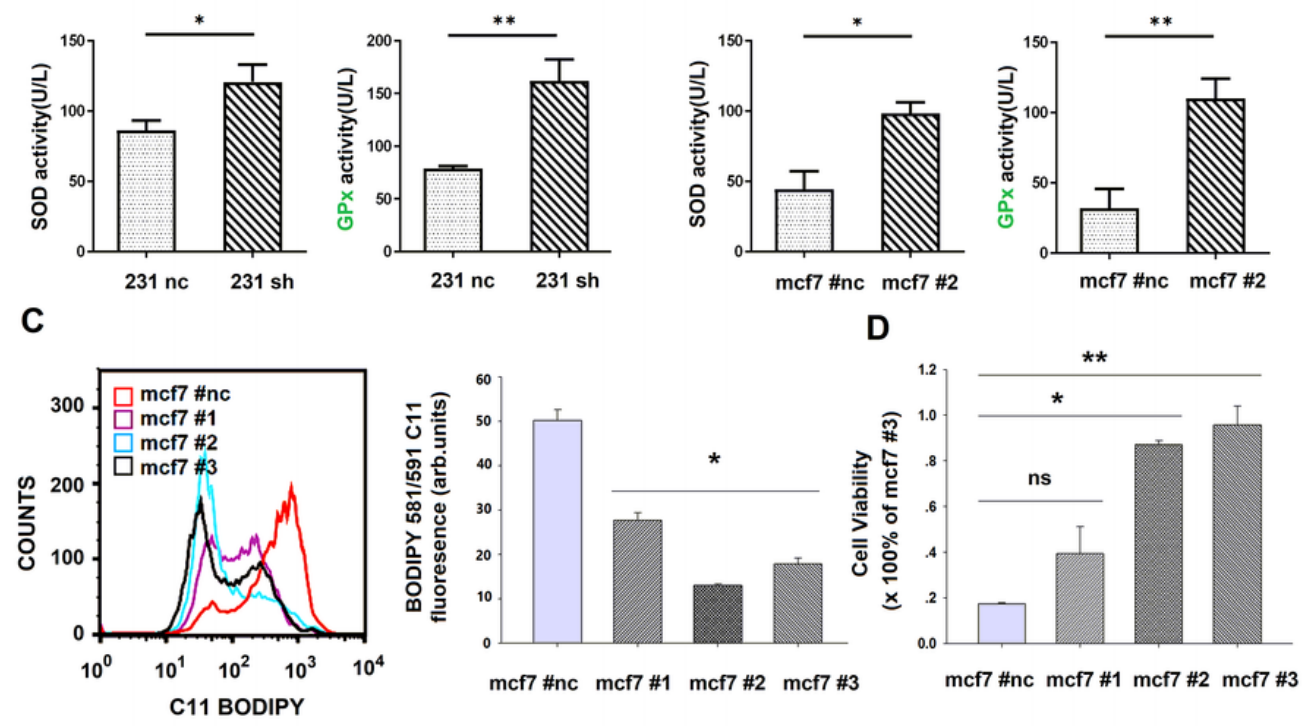

E
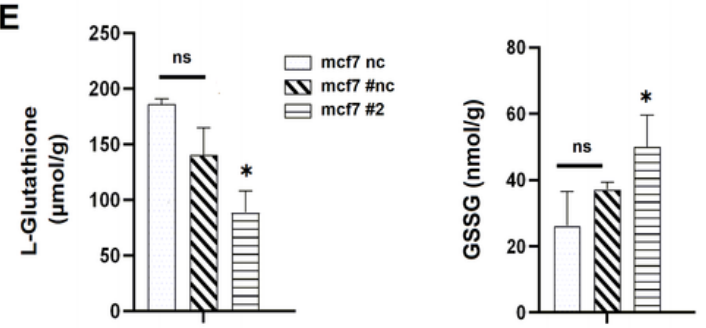

$\mathbf{F}$

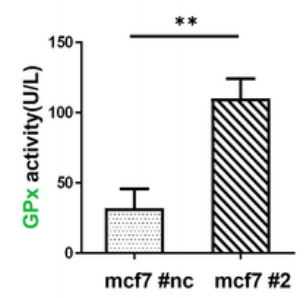

\#nc mcf7 \#2

D
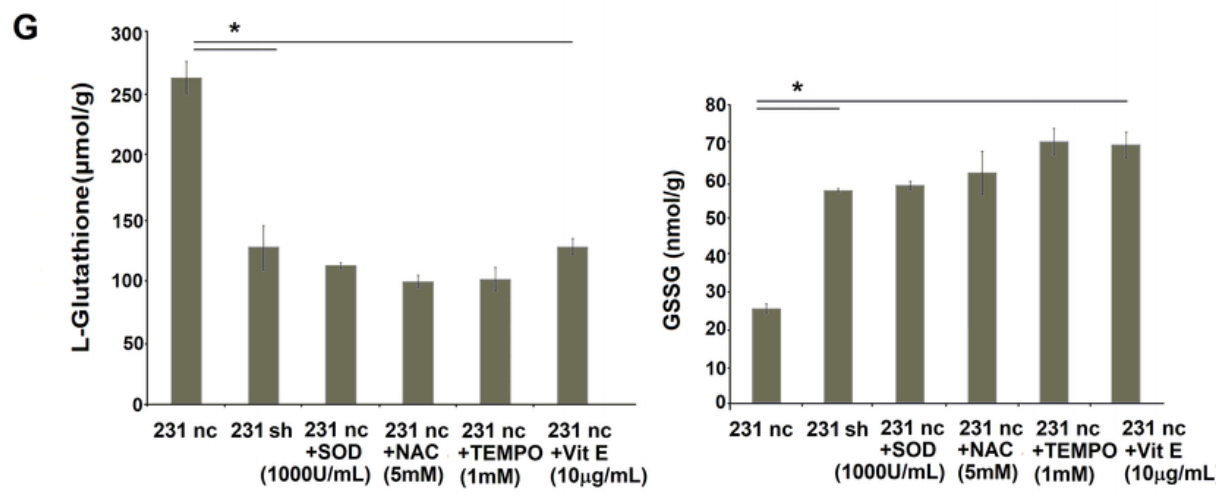

Figure 4

ZEB1 negatively regulates GPX4 contributing to ROS increase in breast cancer cells. A, SOD and GPx activity was detected with sh/siZEB1 in MDA-MB-231 and MCF7 cells. B and D, lipid-ROS intensity and MFI were detected and quantified in siZEB1 MCF7 cells, further cell viability was examined. E, concentrations of L-glutamine and GSSG were detected in different groups in MCF7 cells. F, the schematic of lipid ROS clearance by GPX4 was outlined. G, concentrations of L-glutamine and GSSG were detected in different groups in ZEB1 silenced or ROS scavengers adding MDA-MB-231 cells. Data are represented as means \pm S.E. in triplicate experiments, ${ }^{*} P<0.05$ and ${ }^{*} P<0.01$. 
A

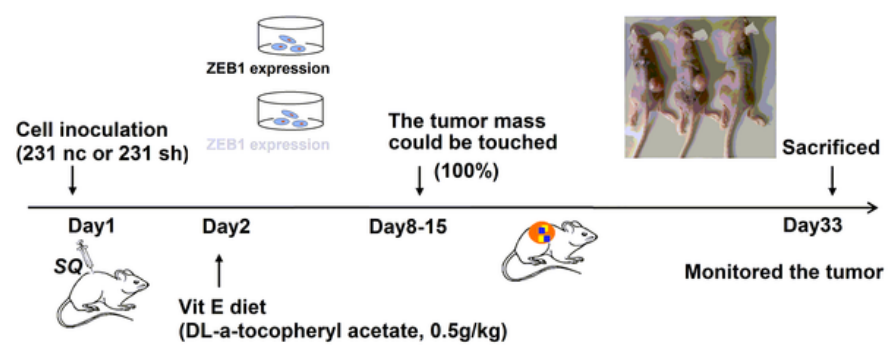

B

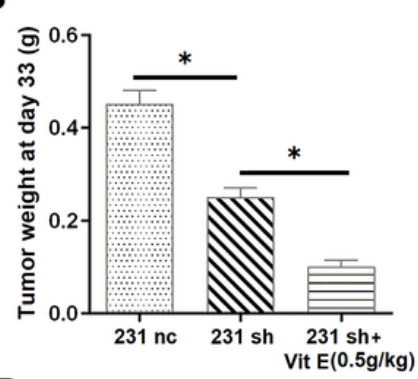

C $\rightarrow 231 \mathrm{nc}$
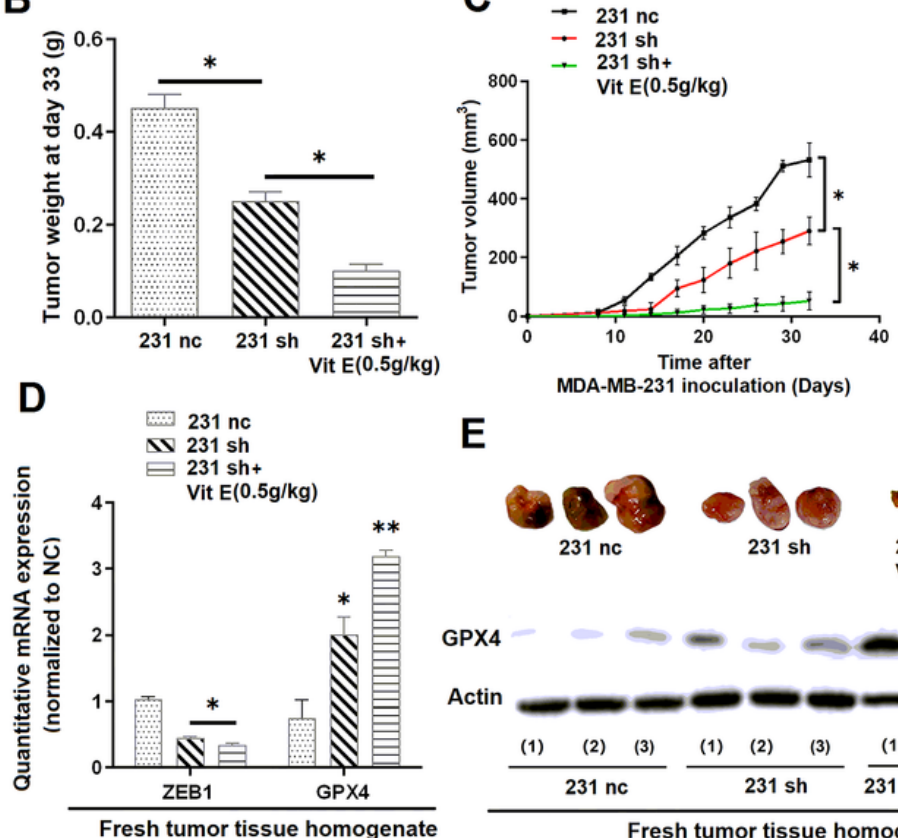

E

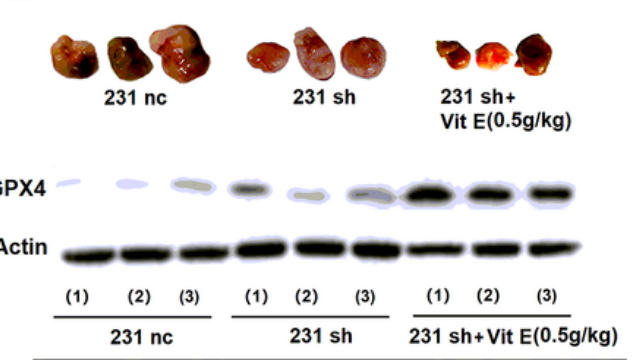

$\mathbf{F}$

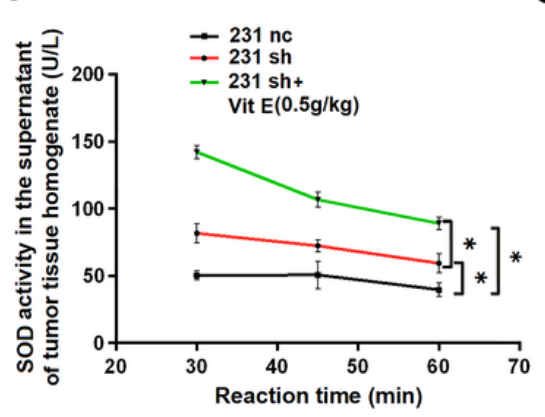

G

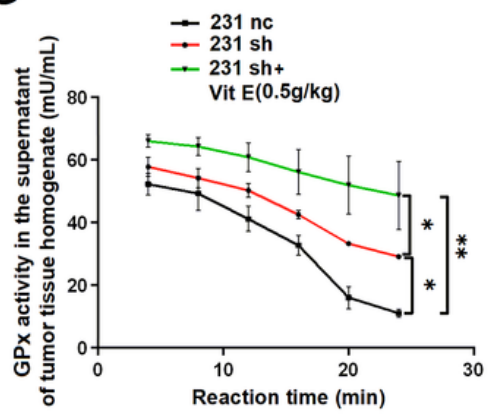

Figure 5

ZEB1 restrains GPX4 expression and vitamin E facilitates GPX4 function in vivo. A, the schematic diagram of tumor formation and drug administration was explained. B and C, statistics of tumor weight and volume in each group. D, ZEB1 and GPX4 mRNA expression in the tumor tissue homogenate were detected by qPCR from different groups. E, representative tumor images from 3 groups of mice $(n=3)$. WB was used to detect GPX4 protein expression in fresh tissues of each tumor. F and G, SOD and GPx activity in fresh tumor tissue homogenate was detected. Data are represented as means \pm S.E., ${ }^{*} P<0.05$ and ${ }^{*}{ }^{*} P<0.01$. 
A
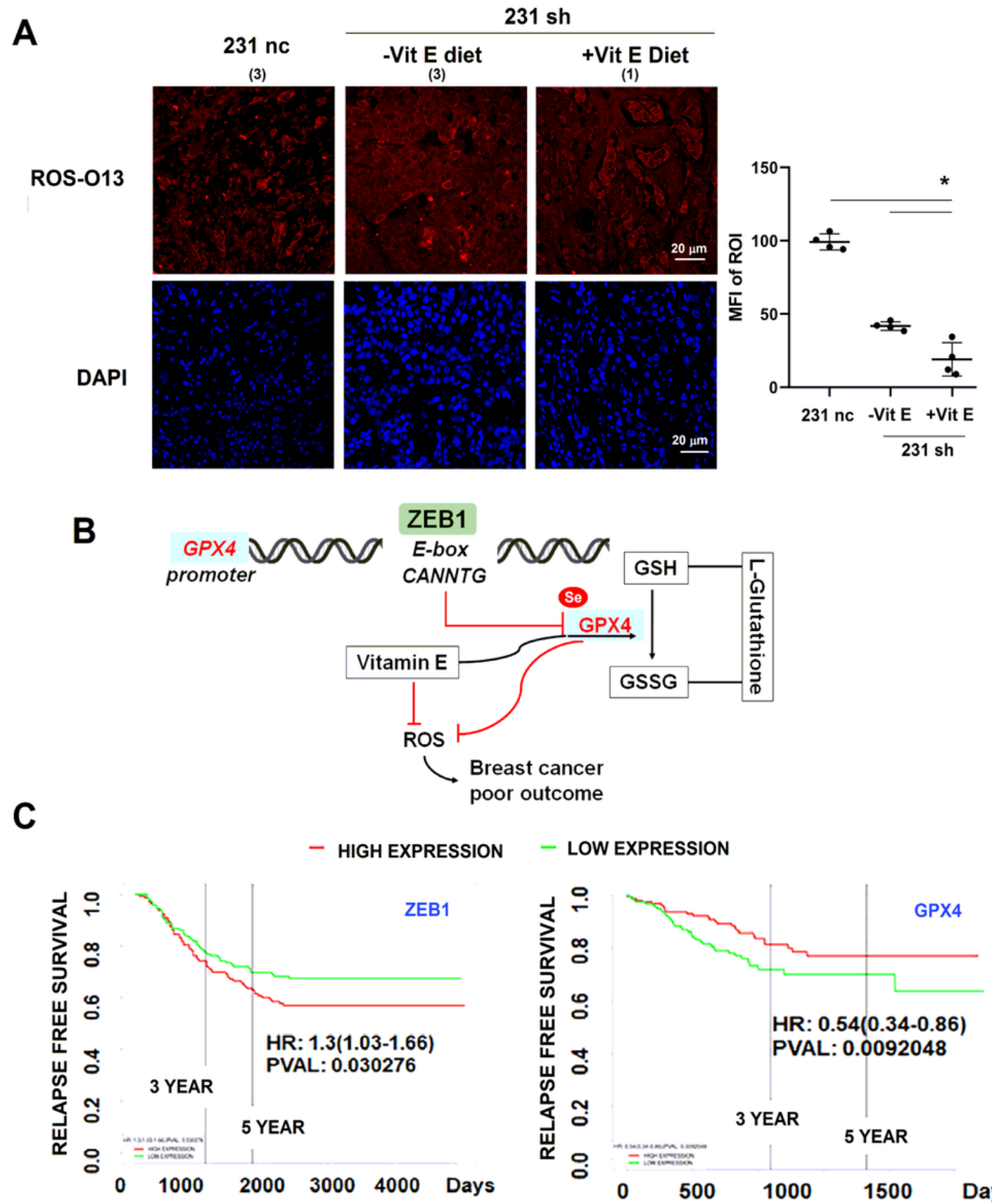

ZEB1

E-box NON

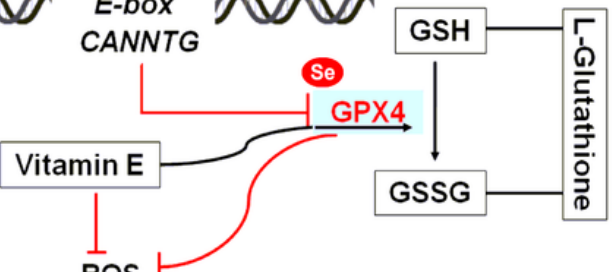

Breast cancer

oor outcome

- LOW EXPRESSION

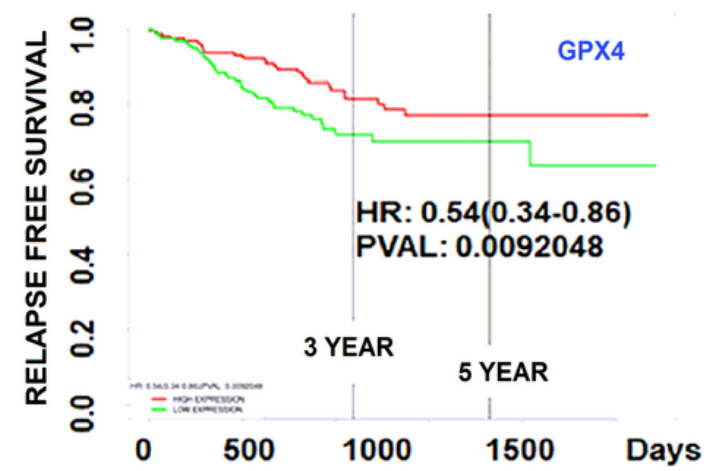

Figure 6

ZEB1 knocked down tumor tissue has decreasing ROS and the clinical significance of mRNA expression of ZEB1 and GPX4. A, frozen sections staining for ROS intensity in fresh tumor tissue (representative number 3 among 231 nc and 231 sh tumor groups, number 1 among 231 sh with Vit E diet group) was shown. MFI of ROI (region of interest, n=4) was quantified, ${ }^{*} \mathrm{P}<0.05$. B, work model was drawn. C, Bioinformatics data from PROGgenev2 database showed relapse-free survival curves of breast cancer patients with high ZEB1 and low GPX4 mRNA expression, which was associated with poor prognosis, ${ }^{\mathrm{P}}<0.05$.

\section{Supplementary Files}

This is a list of supplementary files associated with this preprint. Click to download. 
- Supplementarydata.docx

- NCshZEB1MDAMB231.xIsx

- ROSrelatedupDEGsIDbyZEB1.xIsx

- COHORTofGPX4geneexpression.jpg

- COHORTofZEB1geneexpression.jpg 